\title{
On Paragrassmann Differential Calculus
}

\author{
A.T.Filippov ${ }^{*}$ A.P. Isaev ${ }^{\dagger}$ and A.B.Kurdikov $\stackrel{\ddagger}{ }$ \\ Laboratory of Theoretical Physics, JINR, Dubna, \\ SU-101 000 Moscow, Russia
}

\begin{abstract}
This paper significantly extends and generalizes our previous paper [1]. Here we discuss explicit general constructions for paragrassmann calculus with one and many variables. For one variable, nondegenerate differentiation algebras are identified and shown to be equivalent to the algebra of $(p+1) \times(p+1)$ complex matrices. If $(p+1)$ is prime integer, the algebra is nondegenerate and so unique. We then give a general construction of the many-variable differentiation algebras. Some particular examples are related to the multi-parametric quantum deformations of the harmonic oscillators.
\end{abstract}

*Address until Dec.22, 1992: Yukawa Inst. Theor. Phys., Kyoto Univ., Kyoto 606, Japan e-mail: filippov@jpnyitp.bitnet filippov@jpnyitp.yukawa.kyoto-u.ac.jp

$\dagger$ e-mail: isaev@theor.jinrc.dubna.su

†e-mail: kurdikov@theor.jinrc.dubna.su 


\section{Dedication}

This paper is in memory of Mikhail Konstantinovich Polivanov. One of the authors (A.T.F.) had a privilege to be a friend of him for many and many years. He was not only a distinguished scientist but a true Russian intellectual having deep roots in Russian culture. It is a deep sorrow that we can no more have a talk with him on science, poetry, religion...

\section{Introduction}

Paragrassmann algebras (PGA) are interesting for several reasons. First, they are relevant to conformal field theories [2]. Second, studies of anyons and of topological field theories show the necessity of unusual statistics. These include not only the well-known parastatistics but fractional statistics as well (see, e.g. Refs. [3]). One can also find in recent literature some hints [ [1] at a connection between PGA and quantum groups [5], [6]. Ref. [1] has demonstrated this connection in some detail, showing that some special PGA are closely related to $q$-oscillators [7], [8] and to a differential calculus on quantum hyperplanes [9], [10]. Finally, it looks aesthetically appealing to find a generalization of the Grassmann analysis [11] that proved to be so successful in describing supersymmetry.

Recently, some applications of PGA have been discussed in literature. In Ref. [12] that inspired many other investigations, a parasupersymmetric generalization of quantum mechanics had been proposed. Ref. [13] has attempted at a more systematic consideration of the algebraic aspects of PGA based on the Green ansatz (see, e.g. [14) and introduced, in that frame, a sort of paragrassmann generalization of the conformal algebra. Applications to the relativistic theory of the first-quantized spinning particles have been discussed in [15]. Further references can be found in [1], [16].

The aim of this paper is to construct a consistent generalization of the Grassmann algebra (GA) to a paragrassmann one preserving, as much as possible, those features of GA that were useful in physics applications. A crucial point of our approach is defining generalized derivatives in the paragrassmann variables satisfying natural restriction allowing to construct a differential calculus. As in the previous paper [1], here we mainly concentrate on the algebraic aspects leaving the applications to future publications.

Section 2 treats algebras generated by one paragrassmann variable $\theta, \theta^{p+1}=0$, and a differentiation operator $\partial$. This generalized differentiation coincides with the Grassmann one for $p=1$ and with the standard differentiation when $p \rightarrow$ $\infty$. We construct a most general realization of these algebras and identify a set of nondegenerate ones which are proved to be equivalent. Simplest useful realizations are presented in Section 3.

In Section 4, simplest PGA generated by many variables $\theta_{i}$ and corresponding differentiations $\partial_{i}$ are defined. They obey the nilpotency condition $\theta^{p+1}=0\left(\partial^{p+1}=\right.$ 0 ) where $\theta(\partial)$ is any linear combination of $\theta_{i}\left(\partial_{i}\right)$, and appear to be naturally related to the non-commutative spaces satisfying the commutation relations $\theta_{i} \theta_{j}=$ 
$q_{i j} \theta_{j} \theta_{i}, i<j$ (and similar relations for $\partial_{i} \partial_{j}$ ), where $q_{i j}^{p+1}=1$. These relations once more demonstrate a deep connection between PGA and quantum groups with deformation parameters $q$ being roots of unity.

Section 5 briefly summarizes the results and presents one more relation of our algebras to quantum groups as well as a speculation on possible applications.

\section{Differential Calculus with One Variable}

In Ref. [1] we have considered paragrassmann algebras $\Gamma_{p+1}(N)$ with $N$ nilpotent variables $\theta_{n}, \theta_{n}^{p+1}=0, n=1, \ldots, N$. Some wider algebras $\Pi_{p+1}(N)$ generated by $\theta_{n}$ and additional nilpotent generators $\partial_{n}$ have also been constructed. These additional generators served for defining a paragrassmann differentiation and paragrassmann calculus. The building block for this construction was the simplest algebra $\Pi_{p+1}(1)$. By applying a generalized Leibniz rule for differentiations in the paragrassmann algebra $\Gamma_{p+1}(N)$ we have found two distinct realizations for $\Pi_{p+1}(1)$ closely related to the $q$-deformed oscillators. We have mentioned in [目] that other realizations of the $\Pi_{p+1}(1)$ may be constructed. The aim of this section is to demonstrate this in detail. We shall also show that, under certain conditions, all these realizations are equivalent and one may choose those which are most convenient for particular problems.

Intuitively, paragrassmann algebra $\Pi_{p+1}$ should be understood as some good $p$-generalization of the classical fermionic algebra $\Pi_{2}$

$$
\begin{aligned}
\theta^{2}=0 & =\partial^{2}, \\
\partial \theta+\theta \partial & =1 .
\end{aligned}
$$

By ' $p$-generalization' we mean that (2.2) is to be replaced by

$$
\theta^{p+1}=0=\partial^{p+1}
$$

(it is implied, of course, that $\theta^{p} \neq 0$ and the same for $\partial$ ). So the question is: which generalization of (2.2) might be called 'good'. Many variants have been tried already (see for example [14]). As a rule, they deal with certain symmetric multilinear combinations, like $\theta^{2} \partial+\theta \partial \theta+\partial \theta^{2}$ (for $p=2$ ), and meet with difficulties when commuting $\theta$ and $\partial$.

To find a correct generalization recall that Eq. (2.2) allows to define the Grassmann differential calculus. It shows how to push the differentiation operator $\partial$ to the right of the variable $\theta$. On the other hand, representing $\partial$ and $\theta$ by $2 \times 2$ real matrices, we can make them Hermitian conjugate and thus interpret as annihilation and creation operators. Then Eq. (2.2) is the normal-ordering rule. The second important feature of this relation is that it preserves the Grassmann grading, - 1 for $\partial$ and +1 for $\theta$. In physics terminology, this means that the normal-ordering is not changing the number of 'particles'.

Thus, to construct a generalization of the relation (2.2), we first define a natural grading in the associative algebra generated by $\theta$ and $\partial$ obeying Eq. (2.3),

$$
\operatorname{deg}\left(\theta^{r_{1}} \partial^{s_{1}} \theta^{r_{2}} \partial^{s_{2}} \ldots \theta^{r_{k}} \partial^{s_{k}}\right)=\Sigma r_{i}-\Sigma s_{i}
$$


and denote by $\Pi_{p+1}(l)$ the linear shell of monomials of the degree $l$. Then our basic requirement is:

$$
a \text { set } L^{(l)}=\left\{\theta^{r} \partial^{s}, r-s=l\right\} \text { forms a basis of } \Pi_{p+1}(l) \text {. }
$$

This immediately reduces the range of possible degrees to $0 \leq l \leq p$ and makes all the subspaces $\Pi_{p+1}(l)$ and the entire algebra

$$
\Pi_{p+1}=\oplus_{l=-p}^{p} \Pi_{p+1}(l)
$$

finite-dimensional:

$$
\pi^{l} \equiv \operatorname{dim}\left(\Pi_{p+1}(l)\right)=p+1-|l|, \operatorname{dim}\left(\Pi_{p+1}\right)=(p+1)^{2} .
$$

Then, by applying the assumptions (2.4) and (2.5) to $\partial \theta$, we find that

$$
\partial \theta=b_{0}+b_{1} \theta \partial+b_{2} \theta^{2} \partial^{2}+\ldots+b_{p} \theta^{p} \partial^{p},
$$

where $b_{i}$ are complex numbers restricted by consistency of the conditions (2.3) and (2.7) and by further assumptions to be formulated below. With the aid of Eq. (2.7), any element of the algebra can be expressed in terms of the basis $\theta^{r} \partial^{s}$, i.e. in the normal-ordered form.

A useful alternative set of parameters, $\alpha_{k}$, also fixing the algebra may be defined by

$$
\partial \theta^{k}=\alpha_{k} \theta^{k-1}+(\ldots) \partial,
$$

where the dots denote a polynomial in $\theta$ and $\partial$. This relation is a generalization of the commutation relation for the standard derivative operator, $\partial_{z} z^{k}=k z^{k-1}+z^{k} \partial_{z}$, and we may define the differentiation of powers of $\theta$ by analogy,

$$
\partial\left(\theta^{k}\right)=\alpha_{k} \theta^{k-1}, \alpha_{0} \equiv 0
$$

to be justified later.

By applying Eq. (2.7) to Eq. (2.8) one may derive the recurrent relations connecting these two sets of the parameters:

$$
\begin{aligned}
\alpha_{1} & =b_{0}, \\
\alpha_{2} & =b_{0}+b_{1} \alpha_{1}, \\
\alpha_{3} & =b_{0}+b_{1} \alpha_{2}+b_{2} \alpha_{1} \alpha_{2}, \\
& \cdots \\
\alpha_{k+1} & =\sum_{i=0}^{k} b_{i} \frac{\left(\alpha_{k}\right) !}{\left(\alpha_{k-i}\right) !},
\end{aligned}
$$

where $\left(\alpha_{k}\right) ! \equiv \alpha_{1} \alpha_{2} \cdots \alpha_{k}$. These relations enable us to express $\alpha_{k}$ as a function of the numbers $b_{i}, 0 \leq i \leq k-1$. The first few expressions are

$$
\begin{aligned}
& \alpha_{1}=b_{0}, \quad \alpha_{2}=b_{0} \frac{1-b_{1}^{2}}{1-b_{1}}, \quad \alpha_{3}=b_{0} \frac{1-b_{1}^{3}}{1-b_{1}}+b_{2} b_{0}^{2}\left(1+b_{1}\right), \\
& \alpha_{4}=b_{0} \frac{1-b_{1}^{4}}{1-b_{1}}+b_{2} b_{1} b_{0}^{2}\left(1+b_{1}\right)+b_{0}\left(b_{3}+b_{2} b_{0}\right)\left(1+b_{1}\right) \alpha_{3}, \ldots
\end{aligned}
$$


The inverse operation, deriving $b_{i}$ in terms of $\alpha_{k}$, is well-defined only if all $\alpha_{k} \neq 0$.

The consistency condition mentioned above requires that the parameters must be chosen so as to satisfy the identity

$$
0 \equiv \partial \theta^{p+1}
$$

Taking into account that the second term in Eq. (2.8) vanishes for $k=p+1$ we have $\alpha_{p+1}=0$, with no other restrictions on the parameters $\alpha_{k}$ with $k \leq p$. The corresponding restriction on $p+1$ parameters $b_{i}$ follow from Eq. (2.10),

$$
\alpha_{p+1}\left(b_{0}, \ldots, b_{p}\right) \equiv b_{0}+b_{1} \alpha_{p}+b_{2} \alpha_{p} \alpha_{p-1}+\ldots+b_{p} \alpha_{p} \alpha_{p-1} \cdots \alpha_{2} \alpha_{1}=0,
$$

where the parameters $\alpha_{i}$ are expressed in terms of $b_{i}$. Any admissible set $\{b\}$ determines an algebra $\Pi_{p+1}^{\{b\}}$ with the defining relations (2.3), (2.7). To each algebra $\Pi_{p+1}^{\{b\}}$ there corresponds a set $\{\alpha\}$. A priori, there are no restrictions on $\{\alpha\}$ but, if we wish to treat $\partial$ as a non-degenerate derivative with respect to $\theta$, it is reasonable to require, in addition to (2.5), that

$$
\text { all } \alpha_{k} \neq 0
$$

So let us call a set $\{b\}$ (and corresponding algebra $\Pi_{p+1}^{\{b\}}$ ) non-degenerate, if the condition (2.13) is fulfilled, and degenerate otherwise. As it was already mentioned, in the non-degenerate case the numbers $b_{i}$ are completely determined by the numbers $\alpha_{k}$, so we can use the symbol $\{\alpha\}$ as well as $\{b\}$.

In general, different sets $\{b\}$ determine non-equivalent algebras $\Pi_{p+1}^{\{b\}}$. At first sight, the algebras corresponding to different sets $\{b\}$ look very dissimilar. However, this is not true for the non-degenerate ones. In fact, all non-degenerate algebras $\Pi_{p+1}^{\{b\}}$ are isomorphic to the associative algebra $\operatorname{Mat}(p+1)$ of the complex $(p+1) \times(p+1)$ matrices.

This isomorphism can be manifested by constructing an explicit exact ('fundamental') representation for $\Pi_{p+1}^{\{b\}}$. With this aim, we treat $\theta$ and $\partial$ as creation and annihilation operators (in general, not Hermitian conjugate) and introduce the ladder of $p+1$ states $|k\rangle, k=0,1, \ldots, p$ defined by

$$
\partial|0\rangle=0,|k\rangle \sim \theta^{k}|0\rangle, \quad \theta|k\rangle=\beta_{k+1}|k+1\rangle .
$$

Here $\beta_{k}$ are some non-zero numbers, reflecting the freedom of the basis choice. As $|p+1\rangle=0$, the linear shell of the vectors $|k\rangle$ is finite-dimensional and in the nondegenerate case, when all $\beta_{k} \neq 0 \quad(k=1, \ldots, p)$, its dimension is $p+1$.

Using (2.14) and (2.8) we find

$$
\partial|k\rangle=\left(\alpha_{k} / \beta_{k}\right)|k-1\rangle
$$

Thus the fundamental (Fock-space) representations of the operators $\theta$ and $\partial$ is

$$
\begin{aligned}
\theta_{m n} & =\langle m|\theta| n\rangle=\beta_{n+1} \delta_{m, n+1}, \\
\partial_{m n} & =\langle m|\partial| n\rangle=\left(\alpha_{n} / \beta_{n}\right) \delta_{m, n-1} .
\end{aligned}
$$


It is not hard to see that, for non-zero parameters $\alpha$, the matrices corresponding to $\theta^{m} \partial^{n} \quad(m, n=0 \ldots p)$ form a complete basis of the algebra $\operatorname{Mat}(p+1)$. The isomorphism is established.

Nothing similar occurs for degenerate algebras. To show an evidence against using them in the paragrassmann calculus, consider an extremely degenerate algebra with $\alpha_{k}=0$, so that $b_{0}=b_{2}=\ldots=b_{p}=0, b_{1} \neq 0$. This algebra has nothing to do with $\operatorname{Mat}(p+1)$, and its properties essentially depend on the value of $b_{1}$. It is abelian if $b_{1}=1$; it is a paragrassmann algebra of the type $\Gamma_{p+1}(2)$ if $b_{1}$ is a primitive root of unity (see [1]), and so on. We hope this remark is not sounding like a death sentence on the degenerate algebras. At least, it has to be suspended until further investigation which will probably prove their usefulness in other contexts. However, if we wish to have paragrassmann calculus similar to the Grassmann one, we have to use the nondegenerate algebras.

In Ref. [1], we mentioned that the structure of the algebra $\Gamma_{p+1}$ depend on arithmetic properties of $p+1$ and that this may give certain restrictions on algebras $\Pi_{p+1}$. Here we can make a much stronger statement. Using Eqs. (10) it is easy to prove that if $\alpha_{n+1}=0$ for some positive $n<p$, then $n+1$ is a divisor of $p+1$. It follows that for prime integer values of $p+1$ the condition (13) is satisfied if $\alpha_{1} \neq 0$ (for $\alpha_{1}=0$ all $\alpha_{n}$ must vanish). Thus all nontrivial algebras $\Pi_{p+1}$ are nondegenerate for prime integer $p+1$. It also follows that nonequivalent degenerate algebras $\Pi_{p+1}$ can be classified by divisors of $p+1$ in the following sense. Let $n+1$ is a divisor of $p+1, \alpha_{n+1}=0, \alpha_{m} \neq 0$ for $1 \leq m \leq n$. Then all $\alpha_{m}$ with $m>n+1$ are defined by the obvious periodicity condition, $\alpha_{m+n+1}=\alpha_{m}$, and $b_{m}$ with $0 \leq m \leq n$ are expressed in terms of $\alpha_{m}$. It is easy to show that $\partial^{n+1}=0$ and so the commutation relation (7) is also completely fixed. This analysis can be pushed further but in what follows we restrict our consideration to the nondegenerate algebras.

Thus, two natural requirements (2.5) and (2.13) reduce the range of possible generalizations of the fermionic algebra $\Pi_{2}$ to the unique algebra $\Pi_{p+1}$ that is isomorphic to $\operatorname{Mat}(p+1)$ t. The grading (2.4) in $\Pi_{p+1}$ corresponds to 'along-diagonal' grading in $\operatorname{Mat}(p+1)$. Different non-degenerate algebras $\Pi_{p+1}^{\{b\}}$ are nothing more than alternative ways of writing one and the same algebra $\Pi_{p+1}$. We will call them versions having in mind that fixing the $b$-parameters is analogous to a gauge-fixing (in H. Weyl's usage).

This implies that we will mainly be interested in 'version-covariant' results, i.e. independent on a version choice. Nevertheless, special versions may have certain nice individual features making them more convenient for concrete calculations (thus allowing for simpler derivations of covariant results by non-covariant methods). Several useful versions will be described below. Before turning to this task we end our general discussion with several remarks.

First. The existence of the exact matrix representation (2.16), (2.17) is very useful for deriving version-covariant identities in the algebra $\prod_{p+1}$. For instance, it

\footnotetext{
${ }^{1} \mathrm{H}$. Weyl in his famous book on quantum mechanics had foreseen relevance of these algebras to physics problems. After detailed description of the spin algebras he discussed more general finite algebras and remarked that the finite algebras like those discussed here will possibly appear in future physics. We think it natural to call $\Pi_{p+1}$ the 'finite Weyl algebra' or 'para-Weyl algebra'.
} 
is easy to check that

$$
\begin{aligned}
\left\{\partial, \theta^{(p)}\right\} & =\left(\Sigma \alpha_{k}\right) \theta^{p-1}, \\
\left\{\partial^{p}, \theta^{(p)}\right\} & =\prod \alpha_{k},
\end{aligned}
$$

and to find many other relations. Here we have introduced a useful notation

$$
\left\{\Xi, \quad \Psi^{(l)}\right\}=\Xi \Psi^{l}+\Psi \Xi \Psi^{l-1}+\ldots+\Psi^{l} \Xi
$$

The identities (2.18) generalize those known in the parasupersymmetric quantum mechanics [12].

Second. One may adjust the parameters $\beta_{k}$ to get a convenient matrix representation for $\theta$ and $\partial$. As a rule, we take $\beta_{k}=1$. Note that for the versions with real parameters $\alpha_{k}$, it is possible to choose $\beta_{k}$ so as to have $\theta^{\dagger}=\partial$. We also normalize $\theta$ and $\partial$ so that $\alpha_{1} \equiv b_{0}=1$. This gives a more close correspondence with the Grassmann relation (2.2).

Third. In a given (non-degenerate) version $\Pi_{p+1}^{\{b\}}$ the components of the vector $R_{\{b\}}^{(l)}=\operatorname{col}\left\{\partial^{j} \theta^{i}\right\}_{i-j=l}$ form a basis of the subspace $\Pi_{p+1}(l)$ that is completely equivalent to the original one having the components $L_{\{b\}}^{(l)}=\operatorname{col}\left\{\theta^{i} \partial^{j}\right\}_{i-j=l}$, see Eq. (2.5). Hence, there must exist a non-degenerate matrix $C_{\{b\}}^{(l)} \in \operatorname{mat}\left(\pi^{(l)}, \mathbf{C}\right)$ connecting these two bases,

$$
R_{\{b\}}^{(l)}=C_{\{b\}}^{(l)} \cdot L_{\{b\}}^{(l)}, \quad l=-p, \ldots, p .
$$

The elements of the $C$-matrix are certain functions of $b_{i}$ which are usually not easy to calculate except simple versions. The original commutation relation (2.7) is also included in the system $(2.20)$, for $l=0$.

Quite similarly, two $L$-bases ( $R$-bases) taken in different versions $\{b\}$ and $\left\{b^{\prime}\right\}$ are connected by a non-degenerate matrix $M_{\left\{b b^{\prime}\right\}}\left(N_{\left\{b b^{\prime}\right\}}\right)$, i.e.

$$
\begin{aligned}
& L_{\{b\}}=M_{\left\{b b^{\prime}\right\}} L_{\left\{b^{\prime}\right\}}, \\
& R_{\{b\}}=N_{\left\{b b^{\prime}\right\}} R_{\left\{b^{\prime}\right\}},
\end{aligned}
$$

where the indices $(l)$ are suppressed. The matrices $M_{\left\{b b^{\prime}\right\}}^{(l)}$ (and $N_{\left\{b b^{\prime}\right\}}^{(l)}$ ) belong to $\operatorname{Mat}\left(\pi^{(l)}\right)$ and obey the cocycle relations:

$$
M_{\left\{b b^{\prime}\right\}} M_{\left\{b^{\prime} b\right\}}=1, \quad M_{\left\{b b^{\prime}\right\}} M_{\left\{b^{\prime} b^{\prime \prime}\right\}} M_{\left\{b^{\prime \prime} b\right\}}=1 .
$$

By applying Eq. (2.20), we immediately get the relation

$$
N_{\left\{b b^{\prime}\right\}}=C_{\{b\}} M_{\left\{b b^{\prime}\right\}} C_{\left\{b^{\prime}\right\}}^{-1}
$$

that permits evaluating $C$-matrices for complicated versions once we know them in one version. In particular, Eq. (2.21) tells that the operator $\partial$ in any version can be represented as a linear combination of the operators $\partial, \theta \partial^{2}, \ldots, \theta^{p-1} \partial^{p}$ of any other version. We shall soon see that this, for instance, allows to realize $q$-oscillators in terms of generators $\theta$ and $\partial$ of other versions, and vice versa. 


\section{Versions of the Paragrassmann Calculus}

Now consider some special versions related to the simplest forms of Eq. (2.7).

(0): Primitive Version

Here $b_{1}=\ldots=b_{p-1}=0, b_{p}=-1$, so that $\alpha_{i}=1$,

$$
\left(\partial_{(0)}\right)_{m n}=\delta_{m, n-1}, \quad \partial_{(0)} \theta=1-\theta^{p} \partial_{(0)}^{p} .
$$

This realization of $\partial$ may be called 'almost-inverse' to $\theta$. In the matrix representation (2.16), (2.17) with $\beta_{k}=1$ we have $\theta^{T}=\partial_{(0)}$. This version is the simplest possible but the differential calculus is a fancy-looking thing in this disguise and it is unsuitable for many applications. Still, it has been used in some applications. For example, the operators $\theta$ and $\partial_{(0)}$ for $p=2$ coincide with parafermions in the formulation of the parasupersymmetric quantum mechanics [12].

(1): q-Version, or Fractional Version

Here $b_{1}=q \neq 0, b_{2}=b_{3}=\ldots=b_{p}=0$, so that

$$
\alpha_{i}=1+q+\ldots+q^{i-1}=\frac{1-q^{i}}{1-q} .
$$

The condition $\alpha_{p+1}=0$ tells that $q^{p+1}=1 \quad(q \neq 1)$, while the assumption that all $\alpha_{i} \neq 0$ forces $q=b_{1}$ to be a primitive root, i.e. $q^{n+1} \neq 1, n<p$. Thus in this version $\left(\partial=\partial_{(1)}\right)$

$$
\begin{gathered}
\partial_{(1)} \theta=1+q \theta \partial_{(1)}, \\
\partial_{(1)}\left(\theta^{n}\right)=(n)_{q} \theta^{n-1}, \quad(n)_{q}=\frac{1-q^{n}}{1-q} .
\end{gathered}
$$

These relations were derived in Ref. [1] by assuming that $\partial$ is a generalized differentiation operator, i.e. satisfying a generalized Leibniz rule (a further generalization is introduced below). The derivative $\partial_{(1)}$ is naturally related to the $q$-oscillators ( $q$-derivative) and to quantum algebras (see [1] and references therein). Eq. (3.2) is also extremely convenient for generalizing to Paragrassmann algebras with many $\theta$ and $\partial$.

\section{(2): Almost Bosonic Version}

For this Version

$$
b_{1}=1, \quad b_{2}=\ldots=b_{p-1}=0, \quad b_{p} \neq 0, \text { so that } \alpha_{k}=k .
$$

The condition $\alpha_{p+1}=0$ gives $b_{p}=-\frac{p+1}{p !}$ and thus

$$
\left(\partial_{(2)}\right)_{m n}=n \delta_{m, n-1}, \quad \partial_{(2)} \theta=1+\theta \partial_{(2)}-\frac{p+1}{p !} \theta^{p} \partial_{(2)}^{p} .
$$

As $\partial_{(2)}\left(\theta^{n}\right)=n \theta^{n-1}(n \neq p+1)$, this derivative is 'almost bosonic'.

Let us now discuss the interrelations between $\theta$ and $\partial$. As we have already mentioned, the notation itself hints at treating $\partial$ as a derivative with respect to $\theta$ (see (2.8)). To be more precise, let us represent vectors as functions of $\theta$

$$
|F\rangle=\sum_{k=0}^{p} f_{k}|k\rangle \Leftrightarrow F(\theta)=\sum_{k=0}^{p} f_{k} \theta^{k}
$$


The action of the derivative $\partial$ on this function is defined by (2.14) and (2.15) $\left(\beta_{k}=\right.$ 1),

$$
\partial(1)=0, \partial\left(\theta^{n}\right)=\alpha_{n} \theta^{n-1} \quad(1 \leq n \leq p) .
$$

It is however clear that this derivative does not obey the standard Leibniz rule $\partial(a b)=\partial(a) b+a \partial(b)$.

So consider the following modification of the Leibniz rule [1], 17]

$$
\partial(F G)=\partial(F) \overline{\mathbf{g}}(G)+\mathbf{g}(F) \partial(G) .
$$

The associativity condition (for differentiating $F G H$ ) tells that $\mathbf{g}$ and $\overline{\mathbf{g}}$ are homomorphisms, i.e.

$$
\mathbf{g}(F G)=\mathbf{g}(F) \mathbf{g}(G), \quad \overline{\mathbf{g}}(F G)=\overline{\mathbf{g}}(F) \overline{\mathbf{g}}(G) .
$$

The simplest natural homomorphisms compatible with the relations (3.4), (3.5) and (3.6) are linear automorphisms of the algebra $\Gamma_{p+1}$,

$$
\mathbf{g}(\theta)=\gamma \theta, \quad \overline{\mathbf{g}}(\theta)=\bar{\gamma} \theta
$$

where $\gamma, \bar{\gamma}$ are arbitrary complex parameters and

$$
\alpha_{k}=\frac{\bar{\gamma}^{k}-\gamma^{k}}{\bar{\gamma}-\gamma} .
$$

Now the condition (2.12) yields the equation

$$
\alpha_{p+1}=\frac{\bar{\gamma}^{p+1}-\gamma^{p+1}}{\bar{\gamma}-\gamma}=0
$$

and assuming nondegeneracy requirements $\alpha_{n} \neq 0(n<p+1)$ we conclude that $\bar{\gamma} / \gamma$ must be a primitive $(p+1)$-root of unity. Thus we may formulate another interesting version of the paragrassmann algebra $\Pi_{p+1}$.

$(3): \mathbf{g}-\overline{\mathbf{g}}-$ Version

In this version, the parameters $\alpha_{k}$ are supposed to be given by Eq. (3.8) and we can calculate $b_{i}$ by solving Eq. (2.10):

$$
b_{0}=1, b_{1}=\bar{\gamma}+\gamma-1, b_{2}=(\bar{\gamma}-\bar{\gamma} \gamma+\gamma-1) /(\bar{\gamma}+\gamma), \ldots
$$

Here $\gamma$ and $\bar{\gamma}$ are complex numbers constrained only by the condition that $q=\bar{\gamma} / \gamma$ is a primitive root of unity

$$
\left(\frac{\bar{\gamma}}{\gamma}\right)^{p+1}=1 .
$$

From Eqs. (3.5) and (3.7) one can derive the following operator relations for the automorphisms $\mathbf{g}, \overline{\mathbf{g}}$

$$
\partial \theta-\gamma \theta \partial=\overline{\mathbf{g}}, \quad \partial \theta-\bar{\gamma} \theta \partial=\mathbf{g} .
$$

For the special case $\gamma=(\bar{\gamma})^{-1}=q^{1 / 2}$, redefining $\partial=a, \theta=a^{\dagger}$ allows to recognize in (3.10) the definitions of the $q$-deformed oscillators in the BiedenharnMacFarlane form [7]. Note that Version-(1) can be derived from Version-(3) by 
putting $\bar{\gamma}=q \gamma=1$, (or, $\bar{\gamma}=1 \gamma=q$ ). So we may regard Version-(3) as a generalization of Version-(1). Moreover, it can be shown that, for $p=2$, both Version-(0) and Version-(2) are specializations of Version-(3). However, it is not true for $p>2$ and, in general, the Leibniz rule (3.5) has to be further modified. To find a most general deformed Leibniz rule we slightly change the definition of the $\mathbf{g}-\overline{\mathbf{g}}$-Version.

(4): Generalized Version

Namely, leaving untouched the equations (3.4) and (3.8), we assume that $\gamma$ and $\bar{\gamma}$ are arbitrary parameters not constrained by Eq. (3.9), i.e.

$$
\tilde{\alpha}_{p+1} \equiv \frac{\bar{\gamma}^{p+1}-\gamma^{p+1}}{\bar{\gamma}-\gamma} \neq 0 \text { but } \alpha_{p+1}=0 .
$$

Then the conditions (2.12), (3.4), and (3.8) are only fulfilled if the equations (3.10) are modified as follows:

$$
\begin{aligned}
& \partial \theta-\gamma \theta \partial=\overline{\mathbf{g}}-\frac{\tilde{\alpha}_{p+1}}{\left(\alpha_{p}\right) !} \theta^{p} \partial^{p}, \\
& \partial \theta-\bar{\gamma} \theta \partial=\mathbf{g}-\frac{\tilde{\alpha}_{p+1}}{\left(\alpha_{p}\right) !} \theta^{p} \partial^{p} .
\end{aligned}
$$

Version-(0) may be derived from this version by substituting $\mathbf{g}\left(\theta^{k}\right)=\delta_{k, 0}$ and $\overline{\mathbf{g}}=1$; this means that $\gamma=0, \bar{\gamma}=1$. Equivalently, we may choose $\mathbf{g}=1$ and $\overline{\mathbf{g}}\left(\theta^{k}\right)=\delta_{k, 0}$; then $\gamma=1, \bar{\gamma}=0$. Versions (1) and (3) are reproduced if we put $\tilde{\alpha}_{p+1}=0$, while Version-(2) may be obtained in the limit $\gamma=\bar{\gamma} \rightarrow 1$. Thus, Version-(4) generalizes all versions defined above.

The relations (3.12) dictate a more general modification of the Leibniz rule

$$
\partial(F G)=\partial(F) \overline{\mathbf{g}}(G)+\mathbf{g}(F) \partial(G)+L z(F, G) .
$$

As follows from Eqs. (3.12), the additional term $L z(.,$.$) belongs to the one di-$ mensional space $\{|p\rangle\}$. We suggest to call this term the 'Leibnizean'. Note that the associativity condition for the rule (3.13) requires Eqs. (3.6) and the additional relation

$$
L z(F G, H)+L z(F, G) \overline{\mathbf{g}}(H)=L z(F, G H)+\mathbf{g}(F) L z(G, H) .
$$

Versions (1) and (2) evidently reproduce the Grassmann calculus for $p=1$ while the limit $p \rightarrow \infty$ gives $\mathbf{g}=\overline{\mathbf{g}}=1$ and $L z=0$, thus reproducing the standard calculus in the dimension one. Other versions obeying the conditions $\lim _{p \rightarrow \infty}(L z)=0$ and $\lim _{p \rightarrow \infty}(\mathbf{g}, \overline{\mathbf{g}})=1$ are much more complicated (e.g. $b_{1}=1, b_{2}=\ldots=b_{p-k}=$ $0, b_{p-k+1} \neq 0, \ldots, b_{p} \neq 0$, for some fixed $\left.k \geq 2\right)$.

Summarizing this discussion, we note that in constructing a paragrassmann calculus for many variables we wish to have a generalized Leibniz rule. A most natural generalization must look like

$$
\partial_{i}(F G)=\partial_{j}(F) \overline{\mathbf{g}}_{i}^{j}(G)+\mathbf{g}_{i}^{j}(F) \partial_{j}(G),
$$

where $\overline{\mathbf{g}}$ and $\mathbf{g}$ are some automorphisms and the summation over $j$ is understood. Only Versions (1) and (2) seem to be suitable in this context. 


\section{Paragrassmann Algebras with Many Variables}

Here we present explicit realizations of some paragrassmann algebras $\Pi_{p+1}(N)$ generated by $N$ coordinates $\theta_{i}, \theta_{i}^{p+1}=0(i=1, \ldots, N)$, and $N$ corresponding derivatives $\partial_{i}, \partial_{i}^{p+1}=0$. The simplest (bilinear) algebras can be constructed in Version-(1). Thus consider the algebra $\Pi_{p+1}(1)$ defined by

$$
\partial \theta-q \theta \partial=1, \quad \partial^{p+1}=\theta^{p+1}=0
$$

where $q$ is any primitive $(p+1)$-root of unity The algebra (4.1) was the starting point for considering the fractional para-supersymmetry 18. Our motivation for using this version is its extreme simplicity. Furthermore, it gives bilinear commutation relations for generators of $\Pi_{p+1}(N)$ that are closely related to the definitions of the quantum hyper-plane [5], covariant q-deformed oscillators [8], and differential calculus on the quantum hyperplane [9]. Other versions given by Eq. (2.7) can be considered similarly but they yield non-bilinear multi-paragrassmann algebras (a generic example will be given below).

The automprphsim generator emerging in the generalized Leibniz rule can be written as

$$
\mathbf{g}=\partial \theta-\theta \partial
$$

It is easy to check that

$$
\theta \partial=(\mathbf{g}-1) /(q-1), \partial \theta=(q \mathbf{g}-1) /(q-1),
$$

and

$$
\mathbf{g} \theta=q \theta \mathbf{g}, \quad \mathbf{g} \partial=q^{-1} \partial \mathbf{g} .
$$

Using this operator we define $N$ paragrassmann variables

$$
\theta_{i}=\mathbf{g}^{\rho_{N}^{i}} \otimes \mathbf{g}^{\rho_{N-1}^{i}} \otimes \cdots \otimes \mathbf{g}^{\rho_{i+1}^{i}} \otimes \theta \mathbf{g}^{\rho_{i}^{i}} \otimes \mathbf{g}^{\rho_{i-1}^{i}} \otimes \cdots \otimes \mathbf{g}^{\rho_{1}^{i}}
$$

with the obvious commutation relations

$$
\theta_{i} \theta_{j}=q^{\rho_{i j}} \theta_{j} \theta_{i}, \quad i<j .
$$

We wish to restrict $N(N-1) / 2$ numbers $\rho_{i j}=\rho_{j}^{i}-\rho_{i}^{j}$ so as any linear combination of $\theta_{i}$ is nilpotent,

$$
\left(\sum_{i=0}^{p} c_{i} \theta_{i}\right)^{p+1}=0,
$$

and hence $\theta_{i}$ generate a paragrassmann algebra $\Gamma_{p+1}(N)$. One simple choice is

$$
\rho_{i j}=a_{j}(i<j)
$$

with all $q^{a_{i}}$ being primitive roots of unity. With this choice, all $\theta_{i}$ for $i<j$ acquire the same multiplier $q^{a_{j}}$ in commuting through $\theta_{j}$. So, if Eq. (4.6) is valid for the linear combinations of the first $(j-1)$ thetas, we may apply Eqs. (35), (36) of Ref. [1] and thus prove it to be valid for any number of thetas (provided that all $q^{a_{i}}$ are primitive roots of unity). 
The ansatz (4.4) generalizes the expressions for many thetas obtained in [1] by certain recurrent procedure. It is natural to define the derivatives $\partial_{i}$ by

$$
\partial_{i}=\mathbf{g}^{\sigma_{N}^{i}} \otimes \mathbf{g}^{\sigma_{N-1}^{i}} \otimes \cdots \otimes \mathbf{g}^{\sigma_{i+1}^{i}} \otimes \mathbf{g}^{\sigma_{i}^{i}} \partial \otimes \mathbf{g}^{\sigma_{i-1}^{i}} \otimes \cdots \mathbf{g}^{\sigma_{1}^{i}}
$$

Then the commutation relations for $\partial_{i}$ are $(i<j)$ :

$$
\begin{aligned}
\partial_{i} \partial_{j} & =q^{\sigma_{i j}} \partial_{j} \partial_{i}, \quad \sigma_{i j}=\sigma_{i}^{j}-\sigma_{j}^{i}, \\
\theta_{i} \partial_{j} & =q^{-\sigma_{i}^{j}-\rho_{j}^{i}} \partial_{j} \theta_{i} \\
\theta_{j} \partial_{i} & =q^{-\sigma_{j}^{i}-\rho_{i}^{j}} \partial_{i} \theta_{j} .
\end{aligned}
$$

Here the parameters $\sigma_{i}^{j}$ are to be chosen so that any linear combinations of the derivatives $\partial$ is also nilpotent

$$
\left(\sum_{i=0}^{p} c_{i} \partial_{i}\right)^{p+1}=0 .
$$

Now, to obtain a closed algebra with quadratic commutation relations we have to solve the following problem $(\star)$ :

to express $\partial_{i} \theta_{i}$ as a linear combination of 1 and $\theta_{j} \partial_{j}, i, j=1, \ldots, N$.

It is more convenient to deal with the expressions

$$
\begin{gathered}
\partial_{i} \theta_{i}-q^{\tau_{i}^{i}+1} \theta_{i} \partial_{i}=\mathbf{g}^{\tau_{N}^{i}} \otimes \cdots \otimes \mathbf{g}^{\tau_{i}^{i}} \otimes \cdots \otimes \mathbf{g}^{\tau_{1}^{i}} \equiv \mathbf{g}^{\left\{\tau^{i}\right\}}, \\
\partial_{i} \theta_{i}-q^{\tau_{i}^{i}} \theta_{i} \partial_{i}=\mathbf{g}^{\tau_{N}^{i}} \otimes \cdots \otimes \mathbf{g}^{\tau_{i}^{i}+1} \otimes \cdots \otimes \mathbf{g}^{\tau_{1}^{i}} \equiv \mathbf{g}^{\left\{\tau^{i}\right\}_{+}},
\end{gathered}
$$

where $\tau_{j}^{i}=\rho_{j}^{i}+\sigma_{j}^{i}$. The terms $\theta_{j} \partial_{j}$ can be represented in the form

$$
E_{j} \equiv(q-1) q^{\tau_{j}^{j}} \theta_{j} \partial_{j}=\mathbf{g}^{\tau_{N}^{j}} \otimes \cdots \otimes \mathbf{g}^{\tau_{j}^{j}}(g-1) \otimes \cdots \otimes \mathbf{g}^{\tau_{1}^{j}}=\mathbf{g}^{\left\{\tau^{i}\right\}_{+}}-\mathbf{g}^{\left\{\tau^{i}\right\}}
$$

It is not hard to realize that the problem $(\star)$ is solvable if and only if, for any $i$, there exists a sequence of operators (4.15) producing (4.13) or 4.14) from $1 \equiv \mathbf{g}^{\{0\}}$. To formulate this more rigorously, consider $2 N$ points $\left\{\left\{\tau^{i}\right\},\left\{\tau^{i}\right\}_{+}, i=1 \ldots N\right\}$ and $N$ oriented segments $E_{i}=\left\{\tau^{i}\right\} \rightarrow\left\{\tau^{i}\right\}_{+}$in an $N$-dimensional space. This set of data composes an oriented graph $\mathcal{G}$ that obviously does not contain cycles since all the segments are mutually orthogonal. After these preliminaries, we can formulate the following

$\mathrm{C} \mathrm{r}$ i t e $\mathrm{r}$ i o $\mathrm{n}$ : The problem $(\star)$ is solvable if and only if the correspondent graph $\mathcal{G}$ is connected (and therefore an oriented tree) and contains the point $\{0\}$.

In other words, this means that one can define an equivalence relation $\sim$ on the set $\mathcal{T}=\left\{\{0\},\left\{\tau^{i}\right\},\left\{\tau^{i}\right\}_{+}, i=1 \ldots N\right\}$, so that

$$
\begin{aligned}
& \text { a) } \quad\left\{\tau^{i}\right\} \sim\left\{\tau^{i}\right\}_{+}, \quad i=1 \ldots N, \\
& \text { b) } u=v \Rightarrow u \sim v, \quad \forall u, v \in \mathcal{T} .
\end{aligned}
$$

Then the criterion tells that the entire $\mathcal{T}$ must be a single equivalence class. 
This criterion gives a simple procedure for getting the commutation relations of $\partial_{i}$ and $\theta_{i}$ :

1. Draw an oriented tree with a root $\{0\}$ and $N$ edges;

2. Label the edges by the numbers from 1 to $N$;

3. Find a path from $\{0\}$ to the beginning of the $i$-th edge;

4. Moving along this path, write

$$
\mathbf{g}^{\left\{\tau^{i}\right\}}=1 \pm E_{j_{1}} \pm E_{j_{2}} \ldots
$$

taking ' + ', if the move agrees with the orientation of the edge $j_{a}$ and '-'otherwise;

5. Use the expressions (4.13) and (4.15).

This algorithm exhausts all admissible possibilities. In particular, it proves that all the numbers $\tau_{j}^{i}$ can only b 0 or \pm 1 . Thus, it brings some restrictions on the exponentials $\rho_{j}^{i}$ and $\sigma_{j}^{i}$, though not too strong. There are no direct restrictions on the values of $\rho_{i j}$ or relations between them coming from the criterion. So the last string of the algebra, the commutation relations of $\partial_{i}$ and $\theta_{i}$, is almost independent of the first four ones, (4.5), (4.9 - 4.11). Note that algebras corresponding to the different graphs are non-equivalent, at least at the level of linear combinations.

Let us now present two simplest examples of the paragrassmann algebras $\Pi_{p+1}(N)$.

$$
\text { 1). } \quad \begin{aligned}
\tau_{j}^{i} & =\rho_{j}^{i}+\sigma i_{j}=0 \quad(i \neq j), \\
\tau_{i}^{i} & =0,-1,
\end{aligned}
$$

or, shortly, $\left\{\tau^{i}\right\}=\{0\}$ or $\left\{\tau^{i}\right\}_{+}=\{0\}$. With this choice, the algebra is $(i<j)$ :

$$
\begin{aligned}
\theta_{i} \theta_{j} & =q^{\rho_{i j}} \theta_{j} \theta_{i}, \\
\partial_{i} \partial_{j} & =q^{\rho_{i j}} \partial_{j} \partial_{i}, \\
\theta_{i} \partial_{j} & =q^{-\rho_{i j}} \partial_{j} \theta_{i}, \\
\theta_{j} \partial_{i} & =q^{\rho_{i j}} \partial_{i} \theta_{j}, \\
\partial_{i} \theta_{i}-q^{2 \tau_{i}^{i}+1} \theta_{i} \partial_{i} & =1 .
\end{aligned}
$$

This algebra has been discussed in [1], [10]. The correspondent graph $\mathcal{G}$ is a bunch of $N$ segments coming from (or to) zero point.

$$
\text { 2). } \quad \begin{aligned}
& \tau_{j}^{i}=1, \quad j<i ; \\
& \tau_{j}^{i}=0, \quad j \geq i,
\end{aligned}
$$

or, shortly, $\left\{\tau^{1}\right\}=\{0\},\left\{\tau^{i+1}\right\}=\left\{\tau^{i}\right\}_{+}$. Here we obtain $(i<j)$ :

$$
\begin{aligned}
\theta_{i} \theta_{j} & =q^{\rho_{i j}} \theta_{j} \theta_{i} \\
\partial_{i} \partial_{j} & =q^{\rho_{i j}+1} \partial_{j} \partial_{i}, \\
\theta_{i} \partial_{j} & =q^{-1-\rho_{i j}} \partial_{j} \theta_{i}, \\
\theta_{j} \partial_{i} & =q^{\rho_{i j}} \partial_{i} \theta_{j} \\
\partial_{i} \theta_{i}-q \theta_{i} \partial_{i} & =1+(q-1) \sum_{j=1}^{i-1} \theta_{j} \partial_{j} .
\end{aligned}
$$

This algebra resembles the differential calculus on the quantum hyperplane [9] (see also [10]). The correspondent graph is a chain of $N$ arrows. Algebras of this kind can exist only for even $p$, as mentioned above. 
Concluding this discussion, we would like to formulate some problems related to complete classifying paragrassmann algebras.

1. It is clear that algebras $\Gamma_{p+1}(N)$ with different sets $\left\{a_{i}\right\}$ (see (4.7)) are not equivalent (unless the two sets are proportional). The question is how fully the ansatz (4.7) exhausts all admissible matrices $\rho_{i j}$ in (4.5)? (We suspect that for $N$ large enough it is exhaustive while for smaller $N$ it is not.)

2. An algebra $\Pi_{p+1}(N)$ can be determined by an oriented tree $\mathcal{G}$ together with a suitable set $\left\{a_{i}\right\}$ (or, more generally, $\left\{\rho_{i j}\right\}$ ). Different trees and sets define nonequivalent algebras that cannot be related by any linear transformation of the variables $\theta$ and $\partial$. The question is: can they be related by a non-linear transformation like that connecting the versions of the algebra $\Pi_{p+1}(1)$ ? In other words, can different $\Pi_{p+1}^{\{\mathcal{G}, a\}}(N)$ be considered as versions of a unique algebra $\Pi_{p+1}(N)$ ?

The final remark concerns possible non-bilinear algebras. Our approach can be generalized to arbitrary version with commutation relations (2.7). With this aim, we first introduce a linear automorphism operator $\mathbf{g}$ in the algebra (2.7) that satisfies the commutation relations (4.3) with some $q$, not necessarily a root of unity. Then, for the multi-paragrassmann generators defined as in Eqs. (4.4), (4.8), one can derive the following algebra $(i<j)$ :

$$
\begin{aligned}
\partial_{i} \theta_{i} & =b_{0}+b_{1} \theta_{i} \partial_{i}+b_{2} \theta_{i}^{2} \partial_{i}^{2}+\ldots+b_{p} \theta_{i}^{p} \partial_{i}^{p} \\
\theta_{i} \theta_{j} & =q^{a_{i}} \theta_{j} \theta_{i} \\
\partial_{i} \partial_{j} & =q^{a_{i}} \partial_{j} \partial_{i}, \\
\partial_{i} \theta_{j} & =q^{-a_{i}} \theta_{j} \partial_{i}, \\
\partial_{j} \theta_{i} & =q^{-a_{i}} \theta_{i} \partial_{j} .
\end{aligned}
$$

To satisfy the equations (4.6), (4.12), we have chosen the parameters $\sigma_{i j}=\rho_{i j}=a_{j}$ for $i<j$ and $\sigma_{i j}=\rho_{i j}=-a_{i}$ for $i>j$. The integer numbers $a_{i}$ are restricted by the condition that all $q^{a_{i}}$ are primitive $(p+1)$-roots of unity. The most important feature of this construction is its independence of the version ('version covariance'). This property is of utmost importance in some applications, e.g. in constructing para-Virasoro algebras to be treated in our next paper. Note, however, that the generalized Leibniz rule (3.14) is only satisfied if the $b$-parameters correspond to the $\mathbf{g}-\overline{\mathbf{g}}$-Version. The algebra (4.21) may be further generalized but we will not present these generalizations here. Non-bilinear algebras deserve a separate thorough investigation.

\section{Conclusion}

In this paper, we have given a general construction of the paragrassmann calculus with one variable and have shown that all nondegenerate algebras $\Pi_{p+1}^{\{b\}}$ are equivalent. Still, different versions may be useful in different applications. As has been shown in the last section, constructing algebras with many variables requires simplest versions. Another reason for a separate consideration of different equivalent versions is the following. Our approach to constructing paragrassmann calculus 
with many variables was to preserve the nilpotency property for linear combinations of $\theta_{i}$ and of $\partial_{i}$. Then the commutation relations between different elements are just calculational tools not having any fundamental meaning. However, we may choose a different viewpoint, considering the algebra of commutation relations as a prime object. Then it would be natural to look for transformations preserving the commutation relations.

Let us discuss this viewpoint. It is clear that transformations

$$
\partial_{i} \rightarrow \partial_{i}^{\prime}=t_{i j} \partial_{j}, \quad \theta_{i} \rightarrow \theta_{i}^{\prime}=t_{i j} \theta_{j}
$$

do not preserve the commutation relations (4.5) and (4.9). To preserve these commutation relations (quantum hyperplane relations) we have to consider $t_{i j}$ as generators of the multiparametric quantum group $G L_{q, \rho_{i j}}$. In particular, we have to require

$$
\begin{gathered}
t_{i k} t_{i j}=q^{\rho_{k j}} t_{i j} t_{i k}, \\
\theta_{k} t_{i j}=t_{i j} \theta_{k} .
\end{gathered}
$$

The main paragrassmann identity now looks as

$$
\left(\theta_{i}^{\prime}\right)^{p+1}=\left(\sum_{j=1}^{N} t_{i j} \theta_{j}\right)^{p+1}=0 .
$$

It is clear that

$$
\left(t_{i k} \theta_{k}\right)\left(t_{i j} \theta_{j}\right)=q^{2 \rho_{k j}}\left(t_{i j} \theta_{j}\right)\left(t_{i k} \theta_{k}\right),
$$

and so Eq. (5.2) is fulfilled only if $q^{2 \rho_{i j}}$ are primitive roots of unity. As an example we present the paragrassmann quantum plane defined by $\theta_{i}=\mathbf{g}^{1 / 2} \otimes \cdots \otimes \mathbf{g}^{1 / 2} \otimes$ $\underbrace{\theta \otimes 1 \otimes \cdots \otimes 1}_{i}$. The nilpotency conditions $\left(\theta_{i}\right)^{p+1}=0$ are obviously satisfied and the commutation relations,

$$
\theta_{i} \theta_{j}=q^{1 / 2} \theta_{j} \theta_{i}, \quad(i<j)
$$

are not changed under the transformations (5.1) with $t_{i j} \in G L_{q^{1 / 2}}(N)$. Then it is clear that

$$
\left(t_{i k} \theta_{k}\right)\left(t_{i j} \theta_{j}\right)=q\left(t_{i j} \theta_{j}\right)\left(t_{i k} \theta_{k}\right)
$$

and, if $q$ is primitive root of unity, we obtain that

$$
\left(\theta_{i}^{\prime}\right)^{p+1}=0
$$

Thus, the paragrassmann quantum plane (5.3) may be regarded as a linear space under rotations of the quantum group $G L_{q^{1 / 2}}$.

We hope that this remark shows a deeper connection between PGA and quantum groups than suggested by simpler observations of Ref. [1]. It is quite possible that there exist other relations implicit in works on finite-dimensional representations of quantum groups with a root-of-unity deformation parameter [19]. These representations are very interesting from the mathematical point of view [19] and have recently 
found applications in rational conformal field theories [20]. However, here and now, we wish to emphasize that our motivation and our starting point were quite far from the theory of quantum groups and so the relations to this field were somewhat surprising to us, especially, for general algebras defined by Eq. (7). In fact, our aim was to generalize the Grassmann calculus and to apply the PGA to describing particles with paragrassmann variables, fractional spin and statistics, para-conformal and para-Virasoro algebras, etc. (the last topic is detailly treated in our next paper [21]). Up to now, the relations between PGA and quantum groups, being themselves interesting and beautiful, were not very helpful in these applications. We hope that better understanding the nature of these relations might be useful for physics applications both of quantum groups and of paragrassmann algebras. In this connection, we have to stress that our fairly general construction of the many-variable paragrassmann calculus is probably not the most general one, and complete classifying of nondegenerate algebras $\Pi_{p+1}(N)$ is highly desirable.

One may hope that this eventually will open a way to applications of PGA to systems of many particles with fractional spins and statistics. It is conceivable that these applications are not necessarily restricted to physics in two spatial dimensions and that PGA might be applied to some three-dimensional systems as well, e.g. to quarks inside hadrons. The idea of considering confined quarks as soliton-like quasiparticles has recently attracted some attention, and a model with one-dimensional soliton-like quarks has been treated in detail [22]. A further step in this direction might be to look for anyon-like excitations (vortices) on the surface of the hadron (the border between two phases of the QCD). In this connection, recent results on braid-group analysis of anyons on topologically nontrivial surfaces [23 might be of great interest. Ref. 23 clearly demonstrates that basic facts of the anyon physics can be derived in terms of algebraic, topology-dependent analysis and thus can be applied not only to strictly planar systems. Of course, these remarks are highly speculative but we decide to include them to hint at interesting physics applications of our apparently abstract analysis.

\section{Appendix}

Here we describe parafermions and parabosons [14] in the framework of our approach to the paragrassmann algebras.

\section{Parafermionic Version.}

Parafermionic generators $\theta$ and $\partial\left(\theta^{p+1}=\partial^{p+1}=0\right)$ satisfy the commutation relations [14]

$$
[[\partial, \theta] \theta]=-\rho \theta, \quad[[\partial, \theta] \partial]=\rho \partial .
$$

It is hard to extract the basis for the algebra with these generators, because we can not move all $\partial$ 's to the right-hand side of any monomial $\ldots \partial^{i} \theta^{j} \partial^{k} \theta^{l} \ldots$ Thus, our aim is to find a structure relation (2.7) which is in agreement with (6.1). To do this, we apply the relations (6.1) to the vector $|k\rangle=\left(\theta^{k}\right)$. Then taking into account 
Eqs. (2.8) we derive the following recurrent equations

$$
\begin{array}{r}
\rho=2 \alpha_{n}-\alpha_{n+1}-\alpha_{n-1} \quad, \quad n=1, \ldots, p, \\
\alpha_{0}=\alpha_{p+1}=0 .
\end{array}
$$

The solution is

$$
\alpha_{n}=n \alpha_{1}-\frac{n(n-1)}{2} \rho, \rho=\frac{2 \alpha_{1}}{p} .
$$

Choosing the normalization condition $\alpha_{1}=1$ we have

$$
\rho=2 / p, \quad \alpha_{n}=n(p+1-n) / p .
$$

From Eqs.(2.10) one can find the parameters $b_{i}$ specifying the commutation relation for $\partial$ and $\theta$ (2.7). For first few $b_{i}$ we obtain

$$
b_{0}=1, b_{1}=\frac{p-2}{p}, b_{2}=-\frac{2}{p(p-1)}, b_{3}=-\frac{4}{p(p-1)(p-2)}, \ldots .
$$

\section{Parabosonic Version.}

This Version is specified by the commutation relations [14]

$$
[\{\partial, \theta\} \theta]=\partial \theta^{2}-\theta^{2} \partial=-\rho \theta, \quad[\{\partial, \theta\} \partial]=\theta \partial^{2}-\partial^{2} \theta=\rho \partial .
$$

Now the recurrence equations are

$$
\begin{aligned}
\alpha_{n+1} & =\alpha_{n-1}-\rho, \quad n=1, \ldots, p ; \\
\alpha_{0} & =\alpha_{p+1}=0 .
\end{aligned}
$$

A solution of these equations (for $\rho \neq 0$ ) exists for even $p$ only. With $\alpha_{1}=1$ we obtain

$$
\begin{aligned}
\rho & =2 / p, \\
\alpha_{n} & =-n / p \text { for even } n, \\
\alpha_{n} & =(p+1-n) / p \text { for odd } n .
\end{aligned}
$$

As above, $b_{i}$ are derived by using Eqs. (2.7)

$$
b_{0}=1, b_{1}=-\frac{p+2}{2}, b_{2}=2 \frac{p+1}{p}, b_{3}=4 \frac{p+1}{p(p-2)}, \ldots .
$$

Thus parafermionic and parabosonic algebras can also be defined by the relations (2.3) and (2.7) with an appropriate choice of the parameters $b_{i}$.

\section{Acknowledgments}

A significant part of this work has been done while one of the authors (A.T.F.) visited the Institute of Theoretical Physics of the University of Turin, and the final version has been completed in the Yukawa Institute of Theoretical Physics. A.T.Filippov wishes to express his deep gratitude to V. de Alfaro, K. Nishijima and Y. Nagaoka for arranging the visits and for invaluable support in these hard times. Kind hospitality and the financial support of INFN and of the YITP that made completing this research possible are deeply appreciated. Useful communications with A.D'Adda, M.Caselle, L.Castellani, R.Floreanini, S.Forte, P.Furlan, A.LeClair, M.Mintchev, O.Ogievetsky, V.Spiridonov, and I.Todorov are acknowledged by all the authors. 


\section{References}

[1] A.T. Filippov, A.P. Isaev and A.B. Kurdikov, Mod. Phys. Lett. A7 (1992) 2129.

[2] A.B. Zamolodchikov and V.A. Fateev, Sov. Phys. JETP 62 (1985) 215;

V. Pasquer and H. Saleur, Nucl. Phys. B330(1990) 523.

[3] O. Greenberg, Phys. Rev. D43 (1991) 4111;

C. Aneziris, A.P. Balachandran and D. Sen, Int. J. Mod. Phys. A6 (1991) 4721;

S. Forte, Rev. Mod. Phys. 64 (1992) 193.

[4] A.P. Polychronakos, Mod. Phys. Lett. A5 (1990) 2325;

R. Floreanini and L. Vinet, J. Phys. A23 (1990) L1019.

[5] L.D. Faddeev, N.Yu. Reshetikhin, and L.A. Takhtajan, Algebra i Anal. 1 (1989) 178 (in Russian, English translation: Leningrad Math. J. 1 (1990) 193);

Yu.I. Manin, Preprint CRM-1561, Montreal (1988);

E. Corrigan, D. Fairlie, and P. Fletcher, J. Math. Phys. 31 (1990) 776.

[6] V.G. Drinfeld, Proc. of the Intern. Math. Congr., Berkeley, 1986;

M. Jimbo, Lett. Math. Phys. 11 (1986) 247.

[7] L. Biedenharn, J. Phys. A22 (1989) L873;

A. MacFarlane, J. Phys. A22 (1989) 4581;

T. Hayashi, Comm. Math. Phys. 127 (1990) 72;

H. Ruegg, J. Math. Phys. 31 (1990) 1085.

[8] P. Kulish and E.V. Damaskinsky, J. Phys. A23 (1990) L415;

M. Chaichian, P. Kulish and J. Lukierski, Phys. Lett. 262B (1991) 43.

[9] J. Wess and B. Zumino, Nucl. Phys. (Proc. Suppl.) 18B (1990) 302;

O. Ogievetsky, Preprint MPI-Ph/91-103, Munich, (1991);

B.M. Zupnik, Preprint NIPF-92/01, Tashkent, (1992).

[10] D.B. Fairly and C.K. Zachos, Phys. Lett. B256 (1991) 43.

[11] F.A. Berezin, Introduction into Algebra and Analysis with Anticommuting Variables, Moscow State University Press, 1983 (in Russian);

R. Casalbuoni, Nuovo Cim., A33 (1976) 389.

[12] V.A. Rubakov and V.P. Spiridonov, Mod. Phys. Lett. A3 (1988) 1337;

V.P. Spiridonov, J. Phys. A24 (1991) L529.

[13] S. Durand, R. Floreanini, M.Mayrand and L.Vinet, Phys. Lett. 233B (1989) 158.

[14] Y. Ohnuki and S. Kamefuchi, Quantum Field Theory and Parastatistics, Univ. of Tokyo Press, 1982;

A.B. Govorkov, Sov. J. Part. Nucl. 14 (1983) 520. 
[15] V.D. Gershun and V.I. Tkach, Problems of Nuclear Physics and Cosmic Rays (Kharkov University Press), 23 (1985) 42 (in Russian);

G. Korchemsky, Phys. Lett. 267B (1991) 497.

[16] V.P. Spiridonov, in: Proc. of the Intern. Seminar Quarks-90, Telavi, USSR, May 1990; Eds. V.A.Matveev et al., World Sci., Singapore, 1990;

V.P. Spiridonov, in: Proc. of the 20-th Intern. Conf. on Diff. Geom. Methods in Theor. Phys., New York, USA, June 1991; Eds. S. Catto and A. Rocha, World. Sci., Singapore, 1991.

[17] N. Jacobson, Lie Algebras, Interscience Publ., N.-Y.-London, 1962.

[18] C. Ahn, D. Bernard and A. LeClair, Nucl. Phys. B346 (1990) 409.

[19] C. De Concini and V.G. Kac, Colloque Diximier, Progr. Math. 92, Birkhauser, 1990

E. Date, M. Jimbo, K. Miki and T. Miwa, Publ. RIMS, Kyoto Univ. 27 (1991) 437;

V. Dobrev, Prog. Theor. Phys. Suppl. 102 (1990) 137.

[20] L. Alvarez-Gaume, C. Gomez and G. Sierra, in: Physics and Mathematics of Strings, Eds. L Brink, D. Friedan and A. Polyakov, World Sci., Singapore, 1990.

[21] A.T. Filippov, A.P. Isaev and A.B. Kurdikov, "Paragrassmann extensions of the Virasoro algebra", to be published.

[22] J. Ellis, Y. Frishman and M. Karliner, Nucl. Phys. B382 (1992) 189.

[23] Y. Hatsugai, M. Kohmoto and Young-Shi Wu, Prog. Theor. Phys. Suppl. 107 (1992) 101. 